\title{
Poder del músculo esquelético en la salud y enfermedad
}

\author{
Power of skeletal muscle in health and disease \\ O poder do músculo esquelético na saúde e na doença
}

Sandra Marcela Mahecha Matsudo ${ }^{1 *}$

Recibido: 4 de marzo de 2021. Aceptado para publicación: 21 de abril de 2021. Publicado en línea: 16 de junio de 2021.

https://doi.org/10.35454/rncm.v4n4.288

\section{Resumen}

Las evidencias científicas son contundentes en mostrar que la inactividad física y el comportamiento sedentario tienen efectos perjudiciales en el músculo esquelético, las arterias, el miocardio y las mitocondrias, lo que aumenta el riesgo de enfermedades cardiovasculares, metabólicas, cáncer $y$, por tanto, la mortalidad prematura. El objetivo de esta revisión es describir los mecanismos fisiológicos de las miocinas liberadas por la contracción del músculo esquelético con el ejercicio físico y la aplicación clínica en la salud y enfermedad de niños y adultos. El ejercicio físico regular libera miocinas, que tienen la capacidad de generar efectos fisiológicos autocrinos, paracrinos y endocrinos, que conllevan respuestas asociadas con mejorar el metabolismo del tejido adiposo, hepático, pancreático, vascular, inmunológico, renal, intestinal y óseo, e impactan también la salud cerebral y cognitiva. El efecto de las miocinas en el cerebro explica la mejora en el desempeño cognitivo y en el comportamiento de niños y adolescentes, así como la prevención del deterioro cognitivo asociado con el envejecimiento. Los profesionales de la salud deben usar la prescripción del ejercicio físico en el ámbito clínico para el tratamiento y control de la obesidad, diabetes, enfermedades cardiovasculares, cáncer, enfermedades reumáticas, neuropsiquiátricas, y promover la salud física y mental en la población.

Palabras clave: miocinas, ejercicio, salud, músculo esquelético.

\section{Summary}

Scientific evidence is overwhelming in showing that physical inactivity and sedentary behavior have detrimental effects on skeletal muscle, arterial vessels, myocardium, and mitochondria, increasing the risk of cardiovascular diseases, metabolic, cancer and, consequently, premature mortality. The aim of this review is to describe the physiological mechanisms of myokines released by skeletal muscle contraction with physical exercise and the clinical application in health and disease in children and adults. Regular physical exercise releases myokines that have the ability to generate autocrine, paracrine and endocrine physiological effects, which in turn lead to responses associated with improved adipose, hepatic, pancreatic, vascular, immune, renal, intestinal and bone tissue metabolism, and also impact brain and cognitive health. The effect of myokines on the brain explains the improvement in cognitive performance and behavior in children and adolescents, as well as the prevention of cognitive decline associated with aging. Health professionals should use the prescription of physical exercise in the clinical setting for the treatment and control of obesity, diabetes, cardiovascular diseases, cancer, rheumatic diseases, neuropsychiatric diseases, and to promote physical and mental health in the population.

Keywords: Myokines; Exercise; Health; Skeletal muscle.

\section{Resumo}

As evidências científicas são fortes em mostrar que a inatividade física e o comportamento sedentário têm efeitos prejudiciais no músculo esquelético, nas artérias, no miocárdio e na mitocôndria, aumentando o risco de desenvolver algumas doenças cardiovasculares (DCV), metabólicas, câncer e como consequência a mortalidade prematura. $\mathrm{O}$ objetivo desta revisão é descrever os mecanismos fisiológicos das miocinas liberadas pela contração do músculo esquelético com o exercício físico e a aplicação clínica na saúde e na doença de crianças e adultos. O exercício físico regular libera miocinas que têm a capacidade de gerar efeitos fisiológicos autócrinos, parácrinos e endócrinos que levam a respostas associadas à melhoria do metabolismo do tecido adiposo, hepático, pancreático, vascular, imunológico, renal, intestinal, e ósseo, como também afetam a saúde cerebral e cognitiva. O impacto das miocinas no cérebro explica a melhora no desempenho cognitivo e no comportamento de crianças e adolescentes, bem como a prevenção do declínio cognitivo associado ao envelhecimento. Os profissionais de saúde devem utilizar a prescrição do exercício físico no ambiente clínico para o tratamento e controle da obesidade, diabetes, DCV, câncer, doenças reumáticas e neuropsiquiátricas, e promover a saúde física e mental da população.

Palavras chave: miocinas, exercício, saúde, músculo esquelético. 
Centro de Investigación en Medicina Deporte Ejercicio y Salud, Clínica MEDS. Posgrado, Facultad de Ciencias, Universidad Mayor, Santiago, Chile.
*Correspondencia: Sandra Marcela Mahecha Matsudo. sandra.mahecha@meds.cl

\section{INTRODUCCIÓN}

El comportamiento sedentario, en especial el tiempo de televisión y uso de pantallas, se ha relacionado con efectos negativos en la salud de niños, adolescentes y adultos, que explican, en buena parte, el aumento de la prevalencia de algunas enfermedades crónicas. Los cambios fenotípicos ocasionados por estos comportamientos se relacionan con aumento de peso, adiposidad, presión arterial, lípidos y peor salud cardiometabólica y ósea ${ }^{(1)}$. Los cambios fenotípicos observados en el comportamiento sedentario son disminución del tamaño del músculo esquelético y de la fuerza muscular, menor capacidad del músculo para oxidar carbohidratos y grasas, aumento de la resistencia a la insulina, alteración homeostática del metabolismo celular del músculo esquelético en una determinada carga de trabajo, menor capacidad de vasodilatación de las arterias coronarias, menor gasto cardíaco máximo y menor volumen de eyección.

Los principales efectos de la inactividad física ${ }^{(2)}$, que generan impactos importantes en el riesgo de enfermedades cardiovasculares y metabólicas, así como los efectos benéficos de la actividad física, se describen en la Tabla 1.

Las evidencias científicas de las últimas décadas ${ }^{(3)}$ son contundentes al mostrar que la actividad física regular puede reducir los riesgos de fracturas óseas (66\%), depresión (48\%), deterioro cognitivo (40\%), diabetes tipo 2 (35\%), hipertensión arterial (33\%), mortalidad (30\%), enfermedad coronaria y accidente vascular encefálico (25\%), cáncer de mama $(20 \%)$ y cáncer colorrectal (19\%). El ejercicio físico regular libera miocinas las cuales tienen la capacidad de generar efectos fisiológicos autocrinos, paracrinos y endocrinos que conllevan respuestas asociadas con mejorar el metabolismo del tejido adiposo, hepático, pancreático, vascular, inmunológico, renal, intestinal y óseo, e impactan también la salud cerebral y cognitiva; por tanto, el objetivo de esta revisión narrativa es describir los mecanismos fisiológicos de las miocinas liberadas por la contracción del músculo esquelético con el ejercicio físico y la aplicación clínica en la salud y enfermedad de niños y adultos.

\section{PAPEL DE LAS MIOCINAS EN LA SALUDY ENFERMEDAD}

Las miocinas son péptidos de tipo citocinas, considerados metabolitos liberados por la contracción del músculo esquelético con acciones autocrinas, paracrinas o endocrinas $^{(4)}$. El término "miocina" se usa desde 2003, y aunque se conocen alrededor de 650 moléculas, solo al $5 \%$ se ha identificado claramente su función. Las "ejercinas" se describen como miocinas o factores derivados del músculo esquelético por el ejercicio aeróbico y de resistencia muscular, que tienen la capacidad de afectar otros órganos (Tabla 2). Las miocinas y ejercinas tienen acciones metabólicas no solo en el propio músculo esquelético, sino en el tejido adiposo, hígado, intestino, páncreas, glándulas adrenales, red vascular, piel, sistema inmune, inflamatorio y cerebro, lo que afecta el control del apetito, la cognición, la neurogénesis y el aprendizaje.

Las citocinas producidas por el músculo esquelético más conocidas son clasificadas como $^{(5,6)}$ :

- Miocinas: IL-6, IL-7, IL-15, METRNL, ácido $\beta$-amino isobutírico (BAIBA), factor neurotrófico derivado del cerebro (BDNF), musclina, decorina, PGC1-a, leukemia inhibitory factor (LIF)

- Hepatocinas: FGF21, ANGPTLY, folistatina (FSTL1)

- Osteocinas: osteocalcina, leptina, adiponectina

- Adipocinas: leptina, adiponectina, resistina

- Inmunocitocinas: TNF $\alpha$, IL-1 $\beta$, MCP-1, IL-4, IL-6, IL-10, IL-13, IL-33.

Los efectos positivos de las citocinas producidas por el músculo esquelético en la salud del ser humano se describen en la Tabla 3.

\section{APLICACIÓN CLÍNICA DEL EJERCICIO EN ENFERMEDADES CRÓNICAS DEL ADULTO}

La aplicación clínica de la prescripción del ejercicio aeróbico, de resistencia muscular, combinado y multimodal, supervisado o realizado en casa, se ha descrito en diversas enfermedades y condiciones clínicas ${ }^{(6,7)}$, 
Tabla 1. Efectos fisiológicos perjudiciales del comportamiento sedentario y benéficos de la actividad física y del ejercicio

\begin{tabular}{|c|c|c|}
\hline & Comportamiento sedentario & Actividad física/ejercicio \\
\hline Mitocondria & $\begin{array}{l}\text { - Mutaciones del ADN mitocondrial } \\
\text { - Anormalidades de la cadena de transporte de } \\
\text { electrones } \\
\text { - Aumento de la fisión de mitocondrias } \\
\text { - Reducción del contenido mitocondrial } \\
\text { - Disminución de la respiración }\end{array}$ & $\begin{array}{l}\text { - } \text { Aumento de la degradación de proteínas dañadas y } \\
\text { nueva síntesis de proteínas funcionales } \\
\text { - } \text { Incremento de la expresión de PGC-1a } \\
\text { - } \text { Aumento del contenido de SIRT3 } \\
\text { - } \text { Incremento del volumen mitocondrial }\end{array}$ \\
\hline $\begin{array}{l}\text { Músculo } \\
\text { esquelético }\end{array}$ & $\begin{array}{l}\text { - Aumento de la IL-6 proinflamatoria y la proteína } \\
\text { C-reactiva } \\
\text { - Activación de los sistemas proteolíticos } \\
\text { - Inactivación de la vía PI3K/Akt/mTOR } \\
\text { - Disminución de la masa muscular } \\
\text { - Aumento de la proporción de fibras híbridas por } \\
\text { desregulación en la expresión de isoformas de miosina } \\
\text { de cadena pesada }\end{array}$ & 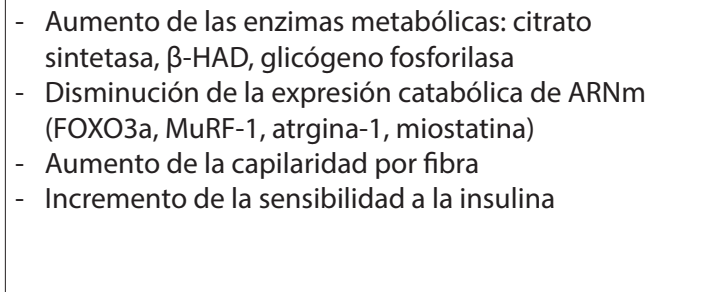 \\
\hline $\begin{array}{l}\text { Músculo } \\
\text { cardíaco }\end{array}$ & $\begin{array}{l}\text { - Aumento del acúmulo de productos finales de glicación } \\
\text { avanzada (AGE), que indican alteración del colágeno } \\
\text { - Aumento de la rigidez ventricular } \\
\text { - Desensibilización de los receptores } \beta \text {-adrenérgicos que } \\
\text { altera las respuestas cronotrópicas e inotrópicas a la } \\
\text { estimulación adrenérgica } \\
\text { - Disminución del SERCA2a, que contribuye al tránsito } \\
\text { del calcio prolongado }\end{array}$ & $\begin{array}{l}\text { - Aumento de la expresión de SERCA2a y de proteína } \\
\text { - Incremento de la fosforilación de treonina-17 que } \\
\text { favorece la recaptura del calcio citoplasmático } \\
\text { - Aumento de las propiedades de contractilidad y } \\
\text { relajación por mejor eficiencia del calcio en la diástole } \\
\text { y sístole } \\
\text { - Disminución de la rigidez del ventrículo izquierdo }\end{array}$ \\
\hline $\begin{array}{l}\text { Arterias de } \\
\text { conducción }\end{array}$ & $\begin{array}{l}\text { - Disminución de la sensibilidad barorrefleja simpática y } \\
\text { - aumento de la activación simpática } \\
\text { - } \text { Aumento del desacoplamiento de la enzima óxido } \\
\text { nítrico sintetasa, disminución de la disponibilidad del } \\
\text { óxido nítrico y aumento del estrés oxidativo } \\
\text { - Remodelación de la matriz extracelular debido a la } \\
\text { degradación de la elastina por metaloproteinasas y } \\
\text { - } \text { formación de AGE } \\
\text { - Disfunción endotelial }\end{array}$ & $\begin{array}{l}\text { - } \text { Menor expresión de transcripción de factor p53 que } \\
\text { - } \text { - Mentá asociado con marcadores de senescencia } \\
\text { - } \\
\text { Disminución de la expresión de nitrotirosina y NADPH } \\
\text { - } \text { oxidasa (prooxidante) } \\
\text { - } \\
\text { (antioxidante) }\end{array}$ \\
\hline
\end{tabular}

Atrgina-1: atrogina 1; $\beta$-HAD: Beta-hydroxyacid dehydrogenase; FOXO3a: forkhead box O3; MuRF-1: muscle RING-finger protein-1; NADPH: nicotinamida adenina dinucleótido fosfato; PGC-1a: proteína 1a coactivadora del receptor activado por el proliferador de peroxisomas; SERCA2a: sarco/retículo endoplásmico $\mathrm{Ca}^{2+}$-ATPasa; SIRT3: Sirtuina 3. Tomada y modificada de ${ }^{(2)}$.

desde el práctico uso del ejercicio aeróbico y de fuerza muscular en pacientes con diabetes tipo $2^{(8)}$, hasta la aplicación clínica en la esteatosis hepática ${ }^{(9)}$, y en el eje microbiota intestinal-cerebro ${ }^{(10)}$. En la literatura médica, el ejercicio físico se considera como una "medicina" ${ }^{(11)}$ por las evidencias existentes de ensayos clínicos aleatorios en al menos 26 enfermedades en siete categorías ${ }^{(12)}$ :

- Musculoesquelético: lumbalgia, lesión de tendón, osteoporosis, osteoartritis y fractura de cadera

- Metabólico: obesidad, diabetes y esteatosis hepática no alcohólica

- Cardiocerebral y vascular: enfermedad arterial coronaria, falla cardíaca crónica y accidente vascular encefálico
- Nervioso: Parkinson, Huntington, Alzheimer, depresión y ansiedad

- Respiratorio: enfermedad pulmonar obstructiva crónica, enfermedad pulmonar intersticial y postrasplante de pulmón

- Urinario: enfermedad renal crónica y postrasplante de riñón

- Cáncer: mama, colon, próstata y pulmón.

Los efectos benéficos del ejercicio físico en la prevención, tratamiento y control de algunas enfermedades se deben a efectos específicos de algunas miocinas en órganos específicos, descritos en la Tabla $4^{(4,6,13)}$. Los principales efectos que llevan a que la actividad física se use en la prevención, tratamiento, control y reha- 
Tabla 2. Miocinas liberadas por la contracción del músculo esquelético de acuerdo con el tipo de ejercicio físico ${ }^{(4)}$

\begin{tabular}{|c|c|c|}
\hline $\begin{array}{c}\text { Aeróbico } \mathbf{y} \\
\text { resistencia }\end{array}$ & $\begin{array}{c}\text { Resistencia y } \\
\text { fuerza muscular }\end{array}$ & $\begin{array}{c}\text { Aeróbico y fuerza } \\
\text { muscular }\end{array}$ \\
\hline BAIBA & CXCL10 & Adiponectina \\
\hline BRINP3 & IL-10 & Apelina \\
\hline CTSB & IL-7 & BDNF \\
\hline CX3CL1 & Lactato & CNTF \\
\hline CXCL12 & Metrnl & FSTK1 \\
\hline FGF-2 & MMP-2 & IGF-1 \\
\hline FGF-21 & & IL-15 \\
\hline Mionectina & & IL-6 \\
\hline Musclina & & Irisina \\
\hline Osteoglicina & & LIF \\
\hline & & Miostatina \\
\hline & & SPARC \\
\hline
\end{tabular}

BAIBA: ácido $\beta$-aminoisobutírico; BDNF: factor neurotrófico derivado del cerebro; BRINP3: gen de la especie Homo sapiens; CNTF: factor neurotrófico ciliar; CTSB: catepsina B; CX3CL1: fractalquina; CXCL10: citocina pequeña inducible B10; CXCL12: proteína inflamatoria de macrófagos 2-alfa; FGF-2: factor 2 del crecimiento del fibroblasto; FGF-21: factor del crecimiento del fibroblasto; FSTL1: Follistatin-like 1; IGF-1: factor de crecimiento insulínico tipo 1 o Somatomedina C: IL: interleucinas; LIF: factor inhibidor de la leucemia; Metrnl: Meteorin-like; MMP-2: metaloproteinasa de matriz 2.

bilitación de las principales enfermedades cardiovasculares se debe a la combinación del conocido efecto antiaterosclerótico, antitrombótico, antiisquémico y antiarrítmico del ejercicio ${ }^{(14-16)}$. Además de estos, también contribuyen los conocidos efectos en el comportamiento, como la disminución del estrés, la ansiedad, la depresión y la hostilidad. Los efectos benéficos del ejercicio aeróbico ocurren no solo en el corazón joven y saludable, sino en las personas mayores y en pacientes con cardiomiopatía congénita y con isquemia miocárdica ${ }^{(17)}$. La Tabla 5 resume los principales efectos benéficos en el sistema cardiovascular y los factores de riesgo asociados con las enfermedades cardiovasculares.

El papel benéfico del ejercicio en la prevención, y como adyuvante del tratamiento y control de los sobrevivientes de cáncer, es bien conocido en adultos. Varios estudios han mostrado efectos benéficos similares en la población pediátrica. Algunos de los efectos fisiológicos y mecanismos inmunometabólicos propuestos que afectan la tumorogénesis, el control del crecimiento de tumores e inhibición de la metástasis se describen en la Tabla $6^{(18-20)}$.

La actividad física y el ejercicio en los sobrevivientes de cáncer se relacionan positivamente con menor fatiga, mejor control del peso y la composición corporal, mejor nivel de fitness cardiorrespiratorio (FCR), salud musculoesquelética y mental, calidad de vida y bienestar, así como disminución del riesgo de mortalidad prematura.

\section{APLICACIÓN CLÍNICA DEL EJERCICIO EN ENFERMEDADES CRÓNICAS EN NIÑOS Y ADOLESCENTES}

El comportamiento sedentario en niños y adolescentes se asocia con efectos negativos en el FCR, la salud cardiometabólica, los comportamientos sociales y la duración del sueño ${ }^{(21,22)}$. Existe una asociación inversa protectora entre el FCR y el síndrome metabólico, la diabetes mellitus tipo 2, la enfermedad del hígado graso no alcohólico y los trastornos de salud mental en la adolescencia. Inclusive existen evidencias de una relación lineal inversa entre el FCR durante la juventud y la mortalidad por todas las causas, y por enfermedades cardiovasculares a lo largo de la vida, así como una asociación positiva con la función cognitiva, la autoestima y la satisfacción con la vida en los jóvenes ${ }^{(21,22)}$. Los beneficios del ejercicio en esta etapa de la vida son ${ }^{(23)}$ :

- Beneficios en preescolares (<6 años)

- Mejor salud ósea

- Reducción del riesgo de aumento excesivo de peso corporal en niños de 3 a 6 años

- No hay evidencias suficientes de efectos benéficos en factores de riesgo cardiometabólico en esta edad

- Beneficios en niños y adolescentes (6-18 años)

- Mejora el FCR (aumenta en 7\%-8 \% el consumo máximo de oxígeno)

- Mejora el fitness muscular, independiente del peso corporal

- Menor aumento de peso y adiposidad

- Asociación positiva con triglicéridos plasmáticos

- Mejora la sensibilidad a la insulina

- Aumenta la masa ósea (1 \%-6 \%) y la estructura y fuerza ósea (3\%-4 \%) 
Tabla 3. Principales efectos fisiológicos de las miocinas, adipocinas, hepatocinas, osteocinas e inmunocinas estimuladas por la contracción del músculo esquelético con el ejercicio

\begin{tabular}{|c|c|}
\hline & Efectos fisiológicos \\
\hline \multicolumn{2}{|l|}{ Miocinas } \\
\hline Ácido a-cetoglutárico & Estimula la hipertrofia muscular y la pérdida de grasa \\
\hline Apelina & $\begin{array}{l}\text { - Controla la presión arterial } \\
\text { - Controla la contractilidad cardíaca } \\
\text { - Minimiza la hipertrofia cardíaca } \\
\text { - Aumenta el consumo de glucosa } \\
\text { - Mejora la función cognitiva }\end{array}$ \\
\hline BAIBA & $\begin{array}{l}\text { - Aumenta el pardeamiento y disminuye la inflamación del TAB } \\
\text { - Incrementa la sensibilidad a la insulina } \\
\text { - Disminuye la lipogénesis y la adipogénesis } \\
\text { - Reduce la resistencia a la insulina en el músculo } \\
\text { - Aumenta la } \beta \text {-oxidación en el músculo e hígado } \\
\text { - Incrementa la función mitocondrial } \\
\text { - Tiene efectos antiinflamatorios sistémicos } \\
\text { - Reduce el acúmulo de grasa } \\
\text { - Atenúa la fibrosis renal } \\
\text { - Preserva la viabilidad de osteocitos } \\
\text { - Disminuye la inflamación hipotalámica }\end{array}$ \\
\hline BDNF & $\begin{array}{l}\text { - Activa la AMPK y la oxidación de lípidos en forma autocrina o paracrina } \\
\text { - Estimula el pardeamiento del TAB } \\
\text { - Regula el metabolismo del músculo esquelético } \\
\text { - Promueve la neurogénesis, el crecimiento y la sobrevivencia de las neuronas del hipocampo, y tiene un } \\
\text { efecto en la memoria y el aprendizaje }\end{array}$ \\
\hline Catepsina-B & - Aumenta el BDNF en el hipocampo \\
\hline CTSB & $\begin{array}{l}\text { - Mejora la función cognitiva de la memoria } \\
\text { - Optimiza la neurogénesis del hipocampo }\end{array}$ \\
\hline $\mathrm{CX} 3 \mathrm{CL} 1$ & - Protege las células $\beta$ contra la acción negativa del TNFa \\
\hline CXCL10 & - Crítico para la angiogénesis dependiente del ejercicio \\
\hline $\begin{array}{l}\text { CXCL12 } \\
\text { (SDF1) }\end{array}$ & $\begin{array}{l}\text { - Protege contra la pérdida de la masa muscular durante la caquexia por cáncer } \\
\text { - Mantiene el crecimiento del músculo }\end{array}$ \\
\hline FGF-2 & - Mejora la formación ósea y el reparo de las fracturas \\
\hline FNDC5 & - Estimula el pardeamiento del TAB \\
\hline IGF-1 & $\begin{array}{l}\text { - Estimula los osteoblastos para la formación ósea } \\
\text { - Mejora la función neuropsiquiátrica }\end{array}$ \\
\hline IL-15 & $\begin{array}{l}\text { - Disminuye el acúmulo de lípidos en los adipocitos } \\
\text { - Reduce la lipogénesis y aumenta la lipólisis } \\
\text { - Incrementa la biogénesis mitocondrial, la oxidación de los ácidos grasos, el consumo de glucosa y la } \\
\text { - sensibilidad a la insulina en el músculo } \\
\text { - Estimula la hipertrofia del músculo esquelético } \\
\text { - } \text { Aumenta el consumo de glucosa y la sensibilidad a la insulina } \\
\text { - Mejora la actividad mitocondrial } \\
\text { - Tiene efectos anabólicos } \\
\text { - Posee efecto de antienvejecimiento de la piel } \\
\text { - Tiene efectos antioxidantes y antiinflamatorios }\end{array}$ \\
\hline
\end{tabular}


Tabla 3. Principales efectos fisiológicos de las miocinas, adipocinas, hepatocinas, osteocinas e inmunocinas estimuladas por la contracción del músculo esquelético con el ejercicio (continuación)

\begin{tabular}{|c|c|}
\hline & Efectos fisiológicos \\
\hline \multicolumn{2}{|l|}{ Miocinas } \\
\hline IL-6 & $\begin{array}{l}\text { - Presenta efectos endócrinos y paracrinos en el músculo esquelético: afecta el consumo de glucosa y la } \\
\text { oxidación de la grasa mediada por la activación de AMPK y aumento de traslocación del transportador } \\
\text { GLUT-4 } \\
\text { - Mejora la lipólisis y la oxidación de grasas } \\
\text { - Disminuye el depósito de grasa visceral y cardíaca } \\
\text { - Apetito: aumenta la proliferación de células pancreáticas y la secreción de GLP-1, retrasa el vaciado } \\
\text { - gástrico y controla la glucosa posprandial, suprime el hambre en el SNC } \\
\text { - Estimula la IL-1 ra y IL-10 que inhiben la producción de TNFa } \\
\text { - Protege la cantidad y función de las células } \beta \text { pancreáticas } \\
\text { - Aumenta la producción de glucosa en el hígado } \\
\text { - Incrementa la sensibilidad a la insulina y la disponibilidad de glucosa para el músculo } \\
\text { - Induce el pardeamiento del TAB } \\
\text { - Tiene efecto antiinflamatorio } \\
\text { - Aumenta la diferenciación de osteoblastos } \\
\text { - Regula el crecimiento muscular } \\
\text { - Mantiene la homeostasis sistémica de glucosa y el metabolismo de lípidos }\end{array}$ \\
\hline Irisina & $\begin{array}{l}\text { - Estimula el pardeamiento del TAB y la termogénesis } \\
\text { - Incrementa la lipólisis del TAB y disminuye el acúmulo de lípidos } \\
\text { - Tiene acción antiinflamatoria y antioxidante } \\
\text { - Aumenta la oxidación de glucosa y ácidos grasos en el músculo esquelético } \\
\text { - Disminuye la glucogenólisis y glucogénesis } \\
\text { - Aumenta la biogénesis mitocondrial } \\
\text { - Disminuye el acúmulo de lípidos en el hígado } \\
\text { - Reduce la apoptosis de las células } \beta \text { pancreáticas } \\
\text { - Aumenta el control del apetito y el gasto energético } \\
\text { - Probablemente estimula la neurogénesis } \\
\text { - Promueve la diferenciación de osteoblastos }\end{array}$ \\
\hline Lactato & $\begin{array}{l}\text { - Promueve la angiogénesis cerebral } \\
\text { - Mejora la función cognitiva del cerebro }\end{array}$ \\
\hline LIF & $\begin{array}{l}\text { - Estimula la proliferación de las células satélite } \\
\text { - Modula la hipertrofia y la regeneración del músculo esquelético } \\
\text { - Estimula la diferenciación de osteoblastos } \\
\text { - Inhibe la diferenciación de adipocitos } \\
\text { - Protege contra la caquexia por cáncer }\end{array}$ \\
\hline METRNL & $\begin{array}{l}\text { - Aumenta el pardeamiento del TAB } \\
\text { - Estimula la expresión de genes de termogénesis de la grasa parda } \\
\text { - Aumenta la sensibilidad a la insulina } \\
\text { - Incrementa la oxidación de ácidos grasos, el consumo de glucosa y la sensibilidad a la insulina en el } \\
\text { - músculo } \\
\text { - Tiene efecto antiinflamatorio }\end{array}$ \\
\hline Mionectina (CTRP15) & $\begin{array}{l}\text { - Mejora el consumo de glucosa } \\
\text { - Estimula la oxidación de ácidos grasos }\end{array}$ \\
\hline Miostatina & $\begin{array}{l}\text { - Regula el crecimiento muscular } \\
\text { - Regula la remodelación ósea } \\
\text { - Atenúa la obesidad } \\
\text { - Disminuye la resistencia a la insulina } \\
\text { - Induce el gasto energético } \\
\text { - Estimula el pardeamiento del TAB }\end{array}$ \\
\hline MMP-2 & $\begin{array}{l}\text { - Aumenta la proliferación y diferenciación celular } \\
\text { - Mantiene la homeostasis celular }\end{array}$ \\
\hline
\end{tabular}


Tabla 3. Principales efectos fisiológicos de las miocinas, adipocinas, hepatocinas, osteocinas e inmunocinas estimuladas por la contracción del músculo esquelético con el ejercicio (continuación)

\begin{tabular}{|c|c|}
\hline & Efectos fisiológicos \\
\hline \multicolumn{2}{|l|}{ Miocinas } \\
\hline Musclina (osteocrina) & $\begin{array}{l}\text { - Atenúa el consumo de glucosa y síntesis de glucógeno } \\
\text { - Incrementa la capacidad del ejercicio } \\
\text { - Protege contra la pérdida de masa muscular por cáncer } \\
\text { - Regula el crecimiento óseo } \\
\text { - Regula la remodelación cerebral } \\
\text { - Controla la presión arterial y la contracción vascular } \\
\text { - Genera biogénesis mitocondrial del músculo }\end{array}$ \\
\hline PGC-1a & - Aumenta la irisina que expresa UCP1 y otros genes brown-fat-like \\
\hline SPARC & $\begin{array}{l}\text { - Regula la remodelación del tejido muscular esquelético } \\
\text { - Inhibe la adipogénesis } \\
\text { - Mejora la tolerancia sistémica a la glucosa }\end{array}$ \\
\hline \multicolumn{2}{|l|}{ Adipocinas } \\
\hline Adiponectina & $\begin{array}{l}\text { - Aumenta la oxidación de ácidos grasos y la sensibilidad a la insulina en el músculo } \\
\text { - Incrementa la oxidación de ácidos grasos, disminuye la lipogénesis y la gluconeogénesis en el hígado } \\
\text { - Induce la acción antiinflamatoria } \\
\text { - Mejora la función de las células } \beta \text { pancreáticas }\end{array}$ \\
\hline IL-10 & $\begin{array}{l}\text { - Induce los efectos antiinflamatorios } \\
\text { - Mejora la tolerancia a la glucosa }\end{array}$ \\
\hline Leptina & $\begin{array}{l}\text { - Disminuye la inflamación hipotalámica } \\
\text { - Aumenta el consumo de glucosa y la oxidación de ácidos grasos libres } \\
\text { - Incrementa la oxidación de ácidos grasos en el hígado } \\
\text { - Reduce el acúmulo de lípidos }\end{array}$ \\
\hline METRN1 & $\begin{array}{l}\text { - Estimula el gasto energético } \\
\text { - Mejora la tolerancia a la glucosa } \\
\text { - Aumenta la termogénesis del tejido adiposo pardo } \\
\text { - Incrementa los niveles de citocinas antiinflamatorias }\end{array}$ \\
\hline \multicolumn{2}{|l|}{ Hepatocinas } \\
\hline ANGPTL4 & $\begin{array}{l}\text { - Aumenta la lipólisis de TAB } \\
\text { - Disminuye la actividad de LPL } \\
\text { - Disminuye la grasa corporal } \\
\text { - Reduce la lipasa pancreática }\end{array}$ \\
\hline FGF21 & $\begin{array}{l}\text { - Aumenta el gasto energético en el tejido adiposo pardo inducido por el SNS } \\
\text { - Incrementa el PGC-1a y el pardeamiento de TAB } \\
\text { - Aumenta la lipólisis del TAB } \\
\text { - Incrementa la oxidación hepática de ácidos grasos } \\
\text { - Disminuye la lipogénesis y la esteatosis hepática } \\
\text { - Aumenta la sensibilidad a la insulina } \\
\text { - Disminuye la ingesta de alimentos ricos en azúcar } \\
\text { - Reduce los niveles de glucosa y triglicéridos }\end{array}$ \\
\hline FSTL1 & $\begin{array}{l}\text { - Aumenta el pardeamiento del TAB } \\
\text { - Estimula la hipertrofia muscular } \\
\text { - Estimula el consumo de glucosa } \\
\text { - Promueve la función de las células endoteliales } \\
\text { - Estimula la revascularización de la lesión cardíaca } \\
\text { - Regula el metabolismo del miocardio } \\
\text { - Mantiene el crecimiento y el desarrollo cardíaco } \\
\text { - Mejora la función de las células } \beta \text { del páncreas } \\
\text { - Protege las células } \beta \text { del páncreas de la apoptosis } \\
\text { - Atenúa la fibrosis y el daño funcional hepático }\end{array}$ \\
\hline
\end{tabular}


Tabla 3. Principales efectos fisiológicos de las miocinas, adipocinas, hepatocinas, osteocinas e inmunocinas estimuladas por la contracción del músculo esquelético con el ejercicio (continuación)

\begin{tabular}{|c|c|}
\hline & Efectos fisiológicos \\
\hline \multicolumn{2}{|l|}{ Osteocinas } \\
\hline BRINP3 (FAM5C) & - Mejora la diferenciación de los osteoblastos \\
\hline CNTF & - Inhibe la diferenciación de los osteoblastos y la formación ósea \\
\hline IL-7 & $\begin{array}{l}\text { - Tiene efectos anabólicos } \\
\text { - Inhibe la formación ósea }\end{array}$ \\
\hline Osteoglicina & $\begin{array}{l}\text { - Promueve el consumo y catabolismo de glucosa y ácidos grasos } \\
\text { - Aumenta los niveles circulantes de IL-6 }\end{array}$ \\
\hline Osteocalcina & $\begin{array}{l}\text { - Incrementa la PGC-1a y la expresión de UCP-1 en el TAB } \\
\text { - Aumenta la sensibilidad a la insulina } \\
\text { - Incrementa la oxidación de ácidos grasos libres y el consumo de glucosa en el músculo } \\
\text { - Aumenta la PGC-1a y la biogénesis mitocondrial en el músculo } \\
\text { - Disminuye la inflamación hepática } \\
\text { - Reduce el acúmulo de lípidos en el hígado } \\
\text { - Disminuye la ingesta de comida por efecto en el SNC } \\
\text { - Aumenta la secreción de insulina } \\
\text { - Incrementa el gasto energético total }\end{array}$ \\
\hline \multicolumn{2}{|l|}{ Inmunocitocinas } \\
\hline IL-10 & - Efecto antinflamatorio \\
\hline IL-13 & - Aumenta la sensibilidad a la insulina \\
\hline IL-33 & - Efecto antinflamatorio \\
\hline
\end{tabular}

ANGPTL4: angiopoyetina 4; AMPK: proteína quinasa activada por AMP; FNDC5: fibronectina tipo III contenido 5; FSTL1: Follistatin-like 1; GLUT-4: proteína transportadora de glucosa tipo 4; PGC-1a: proteína 1a coactivadora del receptor activado por el proliferador de peroxisomas; SNC: sistema nervioso central; SNS: sistema nervioso simpático; SPARC: proteína ácida secretada y rica en cisteína; TAB: tejido adiposo blanco; TNFa: factor de necrosis tumoral alfa; UCP-1: Uncoupling protein one.

Adaptada de ${ }^{(4,5)}$.

- Mejora la cognición: desempeño en pruebas académicas, función ejecutiva, velocidad de procesamiento y memoria (6-13 años)

- Disminuye el riesgo de depresión (6-13 años).

A pesar de que la mayoría de los estudios de la aplicación clínica del ejercicio se realizaron en adultos con enfermedades crónicas, de acuerdo con las evidencias existentes $^{(24)}$, el ejercicio físico regular también tiene efectos beneficios en niños y adolescentes con diagnóstico de algunas enfermedades crónicas:

- Enfermedades respiratorias: en la fibrosis quística se reportaron beneficios en el FCR, en la fuerza muscular y en la calidad de vida. En los pacientes con asma, los efectos incluyen mejora de los síntomas, disminución de la necesidad de consultas de urgencia, menor uso de medicamentos, mejor calidad de vida, menos sibilancias y reactividad bronquial, pero sin efectos en la función pulmonar $y$, por tanto, en la gravedad de la enfermedad.

- Enfermedades cardíacas congénitas: mejora en la capacidad del ejercicio.

- Enfermedades metabólicas: en la obesidad y diabetes tipo 2, el ejercicio es una estrategia fundamental en la prevención y control de estas condiciones, ya que disminuye la adiposidad, mejora el riesgo cardiometabólico y aumenta la masa muscular, el FCR y el metabolismo de la glucosa.

- Enfermedades inflamatorias: en la artritis idiopática juvenil y en la artritis reumatoide se han reportado 
Tabla 4. Efectos de las miocinas en diversos órganos que explican la aplicación clínica del ejercicio en la prevención, tratamiento y control de las enfermedades crónicas $^{(4,6,13)}$

\begin{tabular}{|c|c|c|}
\hline Órgano & Miocinas & Efecto clínico \\
\hline \multirow[t]{8}{*}{$\begin{array}{l}\text { Músculo } \\
\text { esquelético }\end{array}$} & IL-6 & $\begin{array}{l}\text { - Disminuye la inflamación } \\
\text { - Aumenta la atrofia muscular }\end{array}$ \\
\hline & BDNF & $\begin{array}{l}\text { - Incrementa la oxidación de ácidos grasos } \\
\text { - Aumenta la regeneración muscular } \\
\text { - Incrementa la oxidación de los ácidos grasos }\end{array}$ \\
\hline & IL-15 & $\begin{array}{l}\text { - Aumenta el metabolismo de las grasas } \\
\text { - Incrementa la diferenciación de los mioblastos } \\
\text { - Aumenta la masa muscular }\end{array}$ \\
\hline & SPARC & - Incrementa el reparo muscular \\
\hline & FG21 & $\begin{array}{l}\text { - Aumenta la masa muscular } \\
\text { - Incrementa la biogénesis mitocondrial }\end{array}$ \\
\hline & Decorina & $\begin{array}{l}\text { - Aumenta la miogénesis } \\
\text { - Disminuye la atrofia muscular }\end{array}$ \\
\hline & Mionectina & $\begin{array}{l}\text { - Reduce la autofagia } \\
\text { - Aumenta la biogénesis mitocondrial }\end{array}$ \\
\hline & Irisina & $\begin{array}{l}\text { - Aumenta la masa muscular } \\
\text { - Incrementa la hipertrofia muscular } \\
\text { - Aumenta la oxidación de los ácidos grasos }\end{array}$ \\
\hline \multirow{8}{*}{$\begin{array}{l}\text { Tejido } \\
\text { adiposo }\end{array}$} & IL-6 & - Inhibe la producción de citocinas proinflamatorias TNF-a e IL-1b $\beta$ \\
\hline & METRNL & - Respuesta inflamatoria del tejido adiposo que expresa los genes IL-10, TGF $\beta$, IFN- $\gamma$, IL-1 $\beta$ \\
\hline & IL-15 & $\begin{array}{l}\text { - Regulación del depósito de lípidos } \\
\text { - Relación con obesidad y sensibilidad a la insulina }\end{array}$ \\
\hline & FGF-21 & - Regulador metabólico con propiedades antinflamatorias \\
\hline & SPARC & - Limita la habilidad del tejido adiposo para acumular los lípidos en la obesidad \\
\hline & Irisina & - Disminuye la grasa corporal \\
\hline & BAIBA & - Estimula la oxidación de ácidos grasos libres en las mitocondrias de adipocitos \\
\hline & Miostatina & $\begin{array}{l}\text { - Induce los genes de lipólisis y oxidación mitocondrial de ácidos grasos libres en el tejido } \\
\text { adiposo e hígado }\end{array}$ \\
\hline \multirow[t]{5}{*}{ Hígado } & IL-6 & - Aumenta la producción hepática de la glucosa \\
\hline & Mionectina & $\begin{array}{l}\text { - Incrementa el consumo de ácidos grasos de los hepatocitos } \\
\text { - Controla la autofagia celular } \\
\text { - Mantiene la homeostasis de energía }\end{array}$ \\
\hline & BAIBA & - Disminuye el estrés del retículo endoplasmático \\
\hline & FGF-21 & $\begin{array}{l}\text { - Estimula la absorción de la glucosa } \\
\text { - Inhibe la gluconeogénesis }\end{array}$ \\
\hline & Irisina & $\begin{array}{l}\text { - Inhibe la lipogénesis } \\
\text { - Controla la homeostasis de la glucosa } \\
\text { - Balance de la gluconeogénesis/glicogénesis }\end{array}$ \\
\hline \multirow[t]{7}{*}{ Páncreas } & Apelina & $\begin{array}{l}\text { - Mejora la sensibilidad a la insulina y los depósitos de glucosa } \\
\text { - Proliferación de las células } \beta \text { en diabetes tipo } 2 \\
\text { - Disminuye la inflamación y fibrosis }\end{array}$ \\
\hline & CXCL10 & - Correlación con la sensibilidad a la insulina \\
\hline & IL-6 & $\begin{array}{l}\text { - Mejora la proliferación de células } \beta \text { y la secreción de insulina } \\
\text { - Estimula la secreción de GLP-1 } \\
\text { - Mejora la glucemia }\end{array}$ \\
\hline & CX3CL1 & - Regula la secreción de insulina y GLP-1 \\
\hline & FST & - Preserva y protege la función de células $\beta$ \\
\hline & Irisina & - Previene la apoptosis de células $\beta$ \\
\hline & Adiponectina & - Influencia la función y la sobrevivencia de células $\beta$ \\
\hline
\end{tabular}


Tabla 4. Efectos de las miocinas en diversos órganos que explican la aplicación clínica del ejercicio en la prevención, tratamiento y control de las enfermedades crónicas ${ }^{(4,6,13)}$ (continuación)

\begin{tabular}{|c|c|c|}
\hline Órgano & Miocinas & Efecto clínico \\
\hline \multirow[t]{4}{*}{ Hueso } & IGF-1 y FGF-2 & $\begin{array}{l}\text { - Libera los factores osteogénicos } \\
\text { - Diferenciación de osteocitos } \\
\text { - Formación ósea }\end{array}$ \\
\hline & LIF & $\begin{array}{l}\text { - Diferenciación de osteoblastos } \\
\text { - Mantiene el crecimiento del esqueleto en estados patológicos e inflamatorios }\end{array}$ \\
\hline & IL-6, IL-7 y MCP-1 & - Afectan el metabolismo óseo \\
\hline & $\begin{array}{l}\text { IL-15 y MMP-2 } \\
\text { BAIBA } \\
\text { Miostatina } \\
\text { Irisina } \\
\text { Osteoglicina } \\
\text { BRINP3 } \\
\end{array}$ & $\begin{array}{l}\text { - Formación y manutención ósea } \\
\text { - Previene la muerte del osteocito y la pérdida ósea } \\
\text { - Reparo óseo } \\
\text { - Preserva la densidad mineral ósea cortical y trabecular } \\
\text { - Anabolismo óseo } \\
\text { - Diferenciación de los osteoblastos }\end{array}$ \\
\hline \multirow{7}{*}{ Corazón } & Apelina & - Ayuda la función cardíaca y protege contra la hipertrofia \\
\hline & Musclina & - Regula la contracción vascular y presión arterial \\
\hline & FSTL1 & $\begin{array}{l}\text { - Atenúa la formación neointimal, promueve la función de células endoteliales y estimula la } \\
\text { revascularización }\end{array}$ \\
\hline & Dermcidina & - Controla la apoptosis y modula la función y sobrevida de los cardiomiocitos \\
\hline & NDNF & $\begin{array}{l}\text { - Disminuye la apoptosis y aumenta la sobrevivencia de cardiomiocitos posinfarto agudo de } \\
\text { miocardio } \\
\text { - Libera óxido nítrico sintetasa, disminuye la hipertrofia del miocardio y la lesión isquémica }\end{array}$ \\
\hline & Mionectina & $\begin{array}{l}\text { - Disminuye la lesión isquémica } \\
\text { - Protege el corazón de la lesión } \\
\end{array}$ \\
\hline & FSTL1 & $\begin{array}{l}\text { - Estimula los fibroblastos para mejorar el reparo cardíaco } \\
\text { - Disminuye la lesión isquémica }\end{array}$ \\
\hline Riñón & Irisina & $\begin{array}{l}\text { - Mejora la función mitocondrial y el metabolismo de glucosa y ácidos grasos de células renales } \\
\text { - Atenúa el daño y la fibrosis del riñón } \\
\text { - Mejora la función renal }\end{array}$ \\
\hline \multirow[t]{5}{*}{$\begin{array}{l}\text { Control de } \\
\text { apetito }\end{array}$} & BAIBA & $\begin{array}{l}\text { - Inhibe la inflamación hipotalámica; aumenta la sensibilidad central de hormonas } \\
\text { anorexigénicas y estimula la producción de leptina }\end{array}$ \\
\hline & IL-6 & - Efecto anorexigénico en neuronas hipotalámicas y en la secreción de insulina y GLP-1 \\
\hline & Irisina & - Efectos anorexigénicos centrales y mayor gasto de energía \\
\hline & Lactato & - Inhibe la producción de grelina \\
\hline & FGF21 & - Menor ingesta de alimentos azucarados obesogénicos \\
\hline $\begin{array}{l}\text { Sistema } \\
\text { linfático }\end{array}$ & & $\begin{array}{l}\text { - Disminuye el estado proinflamatorio perilinfático, células T, macrófagos y citocinas } \\
\text { proinflamatorias } \\
\text { - Aumenta la función linfática por disminución de estado inflamatorio del tejido subcutáneo } \\
\text { - } \text { Incrementa las corrientes linfáticas y la arquitectura de linfonodos } \\
\text { - Aumenta la migración de las células dendríticas y marcadores endoteliales, lo que mejora el } \\
\text { - transporte de las células inmunitarias } \\
\text { - Disminuye la permeabilidad de los vasos linfáticos } \\
\text { - Aumenta la linfangiogénesis por aumento de VEGF-C/D y VEGFR-3 } \\
\text { - Mejora la densidad de vasos linfáticos y fracción de área en el músculo esquelético } \\
\text { - Aumenta la relación capilar/fibra muscular } \\
\text { - Incrementa el drenaje linfático }\end{array}$ \\
\hline Intestino & & $\begin{array}{l}\text { - Mantiene la integridad de la membrana epitelial y el estado inflamatorio del intestino } \\
\text { - Mejora la homeostasis y la regulación energética, lo que aumenta la diversidad microbiana } \\
\text { - Disminuye los mediadores inflamatorios } \\
\text { - Aumenta las enzimas antioxidantes } \\
\text { - Disminuye la expresión del TNFa en los linfocitos intestinales } \\
\text { - Produce microbiota más diversa, lo que reduce las comunidades bacterianas patogénicas y } \\
\text { aumenta las benéficas }\end{array}$ \\
\hline
\end{tabular}

GLP-1: péptido similar al glucagón tipo 1; IFN- $\gamma$ : interferón $\gamma$; IL-1 $\beta$ : interleucina 1 beta; SPARC: proteína acida secretada y rica en cisteína; TGF $\beta$ : factor de crecimiento transformante beta; VEGF-C/D: factor C y D crecimiento endotelial vascular; VEGFR-3: factor crecimiento endotelial vascular - ligando del tercer receptor. 
Tabla 5. Efectos cardiovasculares del ejercicio aeróbico que contribuyen en la prevención primaria y secundaria de las principales enfermedades cardiovasculares

\begin{tabular}{|c|c|c|}
\hline Factores del riesgo cardiovascular & Vascular & Miocardio y corazón \\
\hline$\uparrow$ fitness cardiorrespiratorio & $\uparrow$ biodisponibilidad de óxido nítrico & $\uparrow$ llenado cardíaco en diástole \\
\hline$\downarrow$ IMC y adiposidad (-5 \%) & $\uparrow$ función endotelial & $\uparrow$ volumen sistólico \\
\hline$\downarrow$ obesidad intraabdominal & $\uparrow$ conductancia capilar & $\uparrow$ función del ventrículo izquierdo \\
\hline$\downarrow$ presión arterial & $\uparrow$ diámetro de las arterias & $\downarrow$ estrés de las paredes \\
\hline$\downarrow$ triglicéridos (-15\%) & $\downarrow$ rigidez arterial & $\downarrow$ demanda de oxígeno \\
\hline$\uparrow \mathrm{HDL}(6 \%)$ & $\downarrow$ resistencia vascular sistémica & $\uparrow$ producción de la energía mitocondrial \\
\hline$\downarrow$ colesterol total $(-5 \%)$ y LDL (-2 \%) & $\downarrow$ estenosis de stents & $\begin{array}{l}\text { Acorta la fase de relajamiento del músculo } \\
\text { cardíaco }\end{array}$ \\
\hline$\downarrow$ relación LDL-C/HDL-C (-5 \%) & $\uparrow$ estabilidad de plaquetas & $\uparrow$ coronarias colaterales \\
\hline$\downarrow$ síndrome metabólico (-37 \%) & $\uparrow$ células progenitoras circulantes & $\uparrow$ reserva coronaria \\
\hline \multirow[t]{5}{*}{$\downarrow$ diabetes tipo 2} & $\uparrow$ formación de vasos y capilares & $\uparrow$ precondicionamiento \\
\hline & $\uparrow$ circulación venosa & $\begin{array}{l}\text { Estimula la expresión de genes en el } \\
\text { corazón }\end{array}$ \\
\hline & $\downarrow$ inflamación endocapilar & $\begin{array}{l}\text { Previene la degeneración y la calcificación } \\
\text { de las válvulas }\end{array}$ \\
\hline & & $\downarrow$ fibrosis cardíaca \\
\hline & & $\begin{array}{l}\text { Revierte la remodelación del ventrículo } \\
\text { izquierdo }\end{array}$ \\
\hline Eléctrico/antiarrítmico & Plaquetas y trombosis & Antiinflamatorio \\
\hline$\uparrow$ tono vagal & $\downarrow$ viscosidad plasmática & $\uparrow$ liberación de miocinas \\
\hline$\uparrow$ variabilidad de la frecuencia cardíaca & $\downarrow$ adhesión plaquetaria & $\downarrow$ proteína C-reactiva (40 \%) \\
\hline $\begin{array}{l}\uparrow \text { tasa de recuperación de la frecuencia } \\
\text { cardíaca }\end{array}$ & $\uparrow$ actividad fibrinolítica & $\uparrow$ IL-6 del músculo \\
\hline$\downarrow$ frecuencia cardíaca en reposo & $\downarrow$ fibrinógeno & $\downarrow$ estrés oxidativo \\
\hline \multicolumn{3}{|l|}{$\uparrow$ expresión de los canales iónicos } \\
\hline \multicolumn{3}{|l|}{$\uparrow$ estabilidad eléctrica } \\
\hline \multicolumn{3}{|l|}{$\downarrow$ actividad adrenérgica } \\
\hline$\downarrow$ arritmias & & \\
\hline
\end{tabular}

HDL-C- colesterol lipoproteínas de alta densidad; IMC: índice de masa corporal; LDL-C: colesterol lipoproteínas de baja densidad. Tomada y modificada de ${ }^{(16)}$.

mejoras en la capacidad funcional, FCR y calidad de vida; sin embargo, lo más importante a destacar es que en la mayoría de las evidencias clínicas existentes no se relatan efectos adversos, por tanto, parece ser una intervención segura en esta población clínica.
- Cáncer: las intervenciones de ejercicio físico realizadas en estos pacientes durante el tratamiento y en los sobrevivientes muestran mejora del FCR y de la fuerza muscular. Otro efecto importante de los ejercicios de fortalecimiento muscular durante el tratamiento con 
Tabla 6. Efectos fisiológicos y mecanismos inmunometabólicos que afectan la tumorogénesis, el control del crecimiento de tumores e inhibición de la metástasis

\begin{tabular}{|l|}
\hline Movilización y redistribución aguda de las células inmunes citotóxicas y NK al tumor \\
\hline Liberación de las miocinas antioncogénicas \\
\hline Inhibición de la formación de nuevas células malignas \\
\hline Liberación de células inmunes como NK, monocitos y linfocitos T por causa de la epinefrina y el shear stress vascular \\
\hline Regulación de las funciones inmunológicas y metabólicas en el microambiente tumoral \\
\hline Disminución de la proporción de células innatas inmunes, macrófagos y MDSC, y aumento de células T CD3+y NK \\
\hline Aumenta la proporción de células T CD8+ y células regulatorias (Treg) y la activación de células T CD8+ (CD69+) \\
\hline Alteración del metabolismo del microambiente tumoral: disminución de la hipoxia e incremento de la vascularización \\
\hline Aumento de la actividad intratumoral de AMPK y disminución de las cinasas AKT, mTOR, PI3K y p42/p44 MAPK \\
\hline Protección de órganos involucrados en la regulación del cáncer, o propensos a malignidad \\
\hline $\begin{array}{l}\text { Mejora en la eficacia antineoplásica de la radioterapia, quimioterapia e inmunoterapia por un efecto sinérgico en el aumento de la } \\
\text { vascularización y mejora de la perfusión sanguínea intratumoral }\end{array}$ \\
\hline Efecto en la médula ósea que mantiene las células inmunes durante las terapias oncológicas \\
\hline Menos toxicidad de los tratamientos con quimioterapia \\
\hline Menos neutropenia, trombocitopenia y linfopenia \\
\hline Mejor disposición de los pacientes para recibir la quimioterapia \\
\hline
\end{tabular}

AKT - Proteína kinasa B; MAPK: proteína cinasa activada por mitógenos; MDSC: myeloid-derived suppressor cells; mTOR: diana de rapamicina en células de mamífero; NK: Natural Killer; T CD: linfocitos T citotóxicos.

quimioterapia en niños con tumores sólidos es el aumento de la fuerza muscular, lo que puede contribuir a disminuir el impacto de la caquexia y de la fatiga en la salud y bienestar de estos pacientes, además que han demostrado ser seguros, cardioprotectores y reducen el tiempo de hospitalización.

\section{EFECTOS QUE TIENE EL EJERCICIO EN EL CEREBRO}

Una de las áreas más fascinantes, y que más ha crecido en investigación en los últimos años, es el efecto de la actividad, el ejercicio y la aptitud física en la salud mental, cognición y desempeño académico de niños y adolescentes ${ }^{(21,25,26)}$. La actividad física y la función cognitiva en escolares de 4 a 18 años incluyen mejoras en las habilidades de percepción, el coeficiente de inteligencia, los logros cognitivos, las pruebas verbales y matemáticas, la memoria, el nivel de desarrollo acadé- mico, el comportamiento en las clases y la concentración en clases de carácter teórico.

Las sesiones agudas de actividad física, de intensidad moderada a vigorosa, han demostrado ejercer efectos benéficos en la cognición, que incluyen la atención y las funciones ejecutivas del cerebro. Dichos beneficios se pueden explicar, en parte, por el papel de las neurotrofinas, factores de crecimiento, catecolaminas y lactato, que influyen en la salud cerebral y la función cognitiva después de la práctica de actividad física.

Una sesión de actividad física aeróbica moderada aumenta la asignación de recursos de atención y mejora el procesamiento cognitivo y la velocidad de clasificación de estímulos, lo cual puede ser un componente muy valioso en el proceso de educación de los escolares. A pesar de la falta de consenso entre los estudios y de los problemas metodológicos asociados, un mejor nivel de FCR puede ser importante para mejorar la salud cerebral y el rendimiento académico de niños y 
adolescentes. La actividad física, el ejercicio, la aptitud física y la participación deportiva pueden mejorar las funciones cognitivas (funciones ejecutivas, atención, memoria, y coeficiente intelectual) que afectan la metacognición (estrategias, conocimiento procedimental y declarativo), lo que impacta el logro o desempeño académico, medido por notas de clase, pruebas estandarizadas o comportamiento en clases ${ }^{(25,26)}$.

Los efectos del ejercicio en la salud cerebral de adultos se han analizado desde su papel en la perfusión cerebral, la neuroplasticidad sináptica, el volumen cerebral, la conectividad y los procesos de neurogénesis y sinaptogénesis, que varían según la intensidad, tipo y duración del ejercicio, así como por el efecto agudo o crónico ${ }^{(27)}$. La mayoría de los estudios demuestra el efecto benéfico del ejercicio en mantener los niveles de hormonas metabólicas, sexuales y de estrés del hipocampo $y$, consecuentemente, en la plasticidad hipocampal, la cognición y la regulación del humor en la adolescencia, la edad adulta y la senescencia ${ }^{(28)}$. Los principales efectos del ejercicio descritos en la literatura en estos aspectos se muestran en la Tabla 7.

La aplicación clínica de los efectos cerebrales del ejercicio se reflejan en la salud mental, cognitiva y neuropsiquiátrica ${ }^{(29)}$, con efectos positivos en condiciones como la depresión pre y posnatal, la anorexia y la bulimia nerviosa, el Binge eating disorder, el estrés postraumático, el abuso de alcohol, las drogas y sustancias ilícitas, la cognición global en esquizofrenia, la depresión en niños y adultos, los síntomas de ansiedad y esquizofrenia, y los trastornos bipolar y de déficit de atención.

El impacto del ejercicio y la condición física en niños y jóvenes con, o en riesgo de, problemas sociales y conductuales incluyen:

- Disminución de la delincuencia y reincidencia

- Reducción del uso de alcohol y aumento de la abstinencia

- Mejora en la responsabilidad

- Reducción de disturbios del comportamiento

- Aumento de la frecuencia a las clases

- Incremento del desempeño académico y vocacional

- Mejora en la relación con los padres y la familia

- Aumento de la autoestima y el autoconcepto

- Incremento en la sensación de bienestar

- Mayor estabilidad emocional

- Mayor nivel de habilidades para la vida

- Disminución de la depresión

- Reducción de la ansiedad

- Disminución de la percepción del estrés.
Tabla 7. Principales efectos fisiológicos del ejercicio en la salud cerebral de los adultos ${ }^{(27,28)}$

\begin{tabular}{|l|}
\hline 1. Aumento del flujo vascular en la arteria cerebral media \\
\hline 2. Estímulo de la angiogénesis cerebral \\
\hline 3. Cambios en la eficacia de las conexiones sinápticas \\
\hline $\begin{array}{l}\text { 4. Aumento en la densidad neuronal y el volumen del } \\
\text { hipocampo }\end{array}$ \\
\hline 5. Mejor conectividad de la funcional neuronal \\
\hline 6. Mayor velocidad de procesamiento \\
\hline 7. Mejor memoria a corto plazo \\
\hline 8. Mejor memoria espacial y verbal \\
\hline 9. Activación funcional de las redes neuronales \\
\hline 10. Mejor eficiencia en las tareas de control ejecutivo \\
\hline $\begin{array}{l}\text { 11. Mejor control ejecutivo de atención y velocidad } \\
\text { perceptual }\end{array}$ \\
\hline 12. Apoya la función regenerativa del cerebro \\
\hline 13. Menor atrofia cerebral \\
\hline 14. Aumenta la sobrevivencia de nuevas neuronas \\
\hline 15. Atenúa la pérdida del sistema nervioso central con la edad \\
\hline 16. Aumenta la integridad de la sustancia blanca cerebral \\
\hline $\begin{array}{l}\text { 17. Incrementa el volumen de la sustancia gris en la región } \\
\text { frontal y temporal }\end{array}$ \\
\hline 18. Expansión de los núcleos cerebrales subcorticales \\
\hline
\end{tabular}

\section{RECOMENDACIÓN AL PROFESIONAL DE SALUD}

El profesional de salud del siglo XXI debe incluir en su quehacer profesional la recomendación de actividad física como forma de promoción de la salud. Las recomendaciones internacionales de actividad física para la salud de niños, adolescentes, y adultos ${ }^{(22)}$ se han adoptado por la Organización Mundial de la Salud (OMS) ${ }^{(30)}$. La recomendación para niños y adolescentes de 5-17 años es limitar el tiempo de comportamientos sedentarios, en especial, el tiempo de pantalla, y realizar al menos 60 minutos diarios de actividad física de intensidad moderada a vigorosa, $y$ actividades de fortalecimiento muscular y óseo 3 días a la semana.

A los mayores de 18 años debe recomendarse sentarse menos y moverse más durante el día, y realizar 
actividad física aeróbica de 150 a 300 minutos por semana, de intensidad moderada o 75-150 de intensidad vigorosa. Para tener mayores beneficios para la salud se pueden hacer más de 300 minutos a la semana de AF moderada, o 150 minutos de vigorosa, o combinación de estas. La recomendación también incluye actividades de fortalecimiento muscular, de intensidad moderada a vigorosa, de grandes grupos musculares, al menos 2 días no consecutivos por semana, y para mejorar la capacidad funcional y evitar caídas, incluir AF multicomponente 3 o más días a la semana.

\section{CONCLUSIÓN}

Los efectos de la liberación de miocinas por la contracción del músculo esquelético por el ejercicio físico son incuestionables, así como su aplicación clínica para la manutención de la salud o para la prevención, tratamiento, control y rehabilitación de las principales enfermedades responsables por la morbimortalidad, que contribuyen en la salud física y mental en cualquier etapa del curso de vida. Todos los profesionales de la salud deberían conocer que el poder del ejercicio físico se explica por los efectos fisiológicos de la contracción muscular esquelética que hacen del músculo el más potente de los órganos endocrinos del ser humano.

\section{PUNTOS CLAVE}

- La inactividad física es un problema mundial de salud pública.

- La actividad física regular disminuye la mortalidad prematura por todas las causas.

- La contracción del músculo esquelético con el ejercicio físico libera sustancias que se comunican con varios sistemas del organismo, lo que proporciona efectos en la prevención, tratamiento y control de diversas enfermedades crónicas en niños, adolescentes y adultos.

- Los beneficios del ejercicio físico ocurren en cualquier edad en la salud mental, el estado cognitivo, el humor y el comportamiento.

Todo profesional de la salud debe incluir la recomendación de actividad y ejercicio físico regular en la infancia, adolescencia y edad adulta, como forma de promoción de salud en personas sanas y con enfermedades crónicas.

\section{Financiación}

El presente estudio no tuvo financiación.

\section{Conflicto de intereses}

El autor declara no tener conflicto de intereses.

\section{Declaración de autoría}

El autor revisó el manuscrito, acuerda ser plenamente responsable de garantizar la integridad y precisión del trabajo, y leyó y aprobó el manuscrito final.

\section{Referencias bibliográficas}

1. Booth FW, Roberts CK, Laye MJ. Lack of exercise is a major cause of chronic diseases. Compr Physiol. 2012,2(2):1143211. doi: 10.1002/cphy.c110025.

2. Lavie CJ, Ozemek C, Carbone S, Katzmarzyk PT, Blair SN. Sedentary behavior, exercise, and cardiovascular health. Circ Res. 2019;124(5):799-815. doi: 10.1161/ CIRCRESAHA.118.312669.

3. Physical Activity Guidelines for Americans [Internet]. U.S. Department of Health and Human Services. $2^{\mathrm{a}}$ edición. 2018. (Consultado el 25 de marzo de 2021). Disponible en: https:// health.gov/sites/default/files/2019-09/Physical_Activity_ Guidelines_2nd_edition.pdf

4. Chen W, Wang L, You W, Shan T. Myokines mediate the cross talk between skeletal muscle and other organs. J Cell Physiol. 2021;236(4):2393-412. doi: 10.1002/jcp.30033.

5. Gonzalez-Gil AM, Elizondo-Montemayor L. The role of exercise in the interplay between myokines, hepatokines, osteokines, adipokines, and modulation of inflammation for energy substrate redistribution and fat mass loss: A review. Nutrients. 2020;12(6):1899. doi: 10.3390/nu12061899.

6. Krogh SMC, Pedersen BK. Muscle-organ crosstalk: the emerging roles of myokines. Endocr Rev. 2020;41(4):594-609. doi: 10.1210/endrev/bnaa016.

7. Pedersen BK, Saltin B. Exercise as medicine - Evidence for prescribing exercise as therapy in 26 different chronic diseases. Scand J Med Sci Sports. 2015;25(3):1-72. doi: 10.1111/ sms.12581.

8. Savikj M, Zierath JR. Train like an athlete: Applying exercise interventions to manage type 2 diabetes. Diabetologia. 2020;63(8):1491-9. doi: 10.1007/s00125-020-05166-9.

9. Thyfault JP, Scott RR. Exercise combats hepatic steatosis: Potential mechanisms and clinical implications. Diabetes. 2020;69(4):517-24. doi: 10.2337/dbi18-0043.

10. Dalton A, Mermier C, Zuhl M. Exercise influence on the microbiome-gut-brain axis. Gut Microbes. 2019;10(5):55568. doi: 10.1080/19490976.2018.1562268.

11. Pedersen BK. The physiology of optimizing health with a focus on exercise as medicine. Annu Rev Physiol. 2019;81:607-27. doi: 10.1146/annurev-physiol-020518-114339.

12. Luan X, Tian X, Zhang H, Huang R, Li N, Chen P, et al. Exercise as a prescription for patients with various disea- 
ses. J Sport Health Sci. 2019;8(5):422-41. doi: 10.1016/j. jshs.2019.04.002.

13. Lee JH, Jun HS. Role of myokines in regulating skeletal muscle mass and function. Front Physiol. 2019;10:42. doi: 10.3389/ fphys.2019.00042.

14. Pinckard K, Baskin KK, Stanford KI. Effects of exercise to improve cardiovascular health. Front Cardiovasc Med. 2019;6:69. doi: 10.3389/fcvm.2019.00069.

15. Franklin BA, Thompson PD, Al-Zaiti SS, Albert CM, Hivert MF, Levine BD, et al. Exercise-related acute cardiovascular events and potential deleterious adaptations following longterm exercise training: placing the risks into perspective-an update: A scientific statement from the American Heart Association. Circulation. 2020;141(13):e705-e36. doi: 10.1161/CIR.0000000000000749.

16. Kasiakogias A, Sharma S. Exercise: The ultimate treatment to all ailments? Clin Cardiol. 2020;43(8):817-26. doi: 10.1002/ clc. 23369 .

17. Moreira JBN, Wohlwend M, Wisløff U. Exercise and cardiac health: Physiological and molecular insights. Nat Metab. 2020;2(9):829-39. doi: 10.1038/s42255-020-0262-1.

18. Christensen JF, Simonsen C, Hojman P. Exercise training in cancer control and treatment. Compr Physiol. 2018;9(1):165205. doi: $10.1002 /$ cphy.c180016.

19. Koelwyn GJ, Zhuang X, Tammela T, Schietinger A, Jone LW. Exercise and immunometabolic regulation in cancer. Nat Metab. 2020;2(9):849-57. doi: 10.1038/s42255-020-00277-4.

20. Sitlinger A, Brander DM, Bartlett DB. Impact of exercise on the immune system and outcomes in hematologic malignancies. Blood Adv. 2020;4(8):1801-11. doi: 10.1182/bloodadvances.2019001317.

21. Bangsbo J, Krustrup P, Duda J, Hillman C, Andersen L, Weiss $\mathrm{M}$, et al. The Copenhagen Consensus Conference 2016: Children, youth, and physical activity in schools and during leisure time. Br J Sports Med. 2016;50(19):1177-8. doi: 10.1136/bjsports-2016-096325.

22. Raghuveer G, Hartz J, Lubans DR, Takken T, Wiltz JL, Mietus-Snyder M, et al. Cardiorespiratory fitness in youth: An important marker of health: A scientific statement from the
American Heart Association. Circulation. 2020;142(7):e10118. doi: 10.1161/CIR.0000000000000866.

23. Piercy KL, Troiano RP, Ballard RM, Carlson SA, Fulton JE, Galuska DA, et al. The physical activity guidelines for Americans. JAMA. 2018;320(19):2020-8. doi: 10.1001/ jama.2018.14854.

24. West SL, Banks L, Schneiderman JE, Caterini JE, Stephens S, White G, et al. Physical activity for children with chronic disease; a narrative review and practical applications. BMC Pediatr. 2019;19(1):12. doi: 10.1186/s12887-018-1377-3.

25. Álvarez-Bueno C, Hillman $\mathrm{CH}$, Cavero-Redondo I, SánchezLópez M, Pozuelo-Carrascosa DP, Martínez-Vizcaíno V. Aerobic fitness and academic achievement: A systematic review and meta-analysis. J Sports Sci. 2020;38(5):582-89. doi: 10.1080/02640414.2020.1720496.

26. Hillman $\mathrm{CH}$, Logan NE, Shigeta TT. A review of acute physical activity effects on brain and cognition in children. Transl J Am Coll Sports Med. 2019;4(17):132-6. doi: 10.1249/ TJX.0000000000000101.

27. Cabral DF, Rice J, Morris TP, Rundek T, Pascual-Leone A, Gomes-Osman J. Exercise for brain health: An investigation into the underlying mechanisms guided by dose. Neurotherapeutics. 2019;16(3):580-99. doi: 10.1007/ s13311-019-00749-w.

28. Bettio LEB, Thacker JS, Rodgers SP, Brocardo PS, Christie BR, Gil-Mohapel J. Interplay between hormones and exercise on hippocampal plasticity across the lifespan. Biochim Biophys Acta Mol Basis Dis. 2020;1866(8):165821. doi: 10.1016/j. bbadis.2020.165821.

29. Ashdown-Franks G, Firth J, Carney R, Carvalho AF, Hallgren $\mathrm{M}$, Koyanagi A, et al. Exercise as medicine for mental and substance use disorders: A meta-review of the benefits for neuropsychiatric and cognitive outcomes. Sports Med. 2020;50(1):151-70. doi: 10.1007/s40279-019-01187-6.

30. Bull FC, Al-Ansari SS, Biddle S, Borodulin K, Buman MP, Cardon G, et al. World Health Organization 2020 guidelines on physical activity and sedentary behavior. Br J Sports Med. 2020;54(24):1451-62. doi: 10.1136/bjsports-2020-102955. 